\title{
Gastric Lipase Measurement
}

National Cancer Institute

\section{Source}

National Cancer Institute. Gastric Lipase Measurement. NCI Thesaurus. Code C117840.

The determination of the amount of gastric lipase present in a sample. 